\title{
Conserved $\mathrm{Cx}_{\mathrm{n}} \mathrm{C}$ Motifs in Kaposi's Sarcoma-Associated Herpesvirus ORF66 Are Required for Viral Late Gene Expression and Are Essential for Its Interaction with ORF34
}

\author{
(D) Allison L. Didychuk, ${ }^{a}$ Angelica F. Castañeda, ${ }^{a}$ Lola O. Kushnir, ${ }^{b}$ Carolyn J. Huang, ${ }^{b}$ Britt A. Glaunsingera,b,c \\ aDepartment of Plant and Microbial Biology, University of California, Berkeley, California, USA \\ bDepartment of Molecular and Cell Biology, University of California, Berkeley, California, USA \\ cHoward Hughes Medical Institute, Berkeley, California, USA
}

\begin{abstract}
Late gene transcription in the beta- and gammaherpesviruses depends on a set of virally encoded transcriptional activators (vTAs) that hijack the host transcriptional machinery and direct it to a subset of viral genes that are required for completion of the viral replication cycle and capsid assembly. In Kaposi's sarcomaassociated herpesvirus (KSHV), these vTAs are encoded by ORF18, ORF24, ORF30, ORF31, ORF34, and ORF66. Assembly of the vTAs into a complex is critical for late gene transcription, and thus, deciphering the architecture of the complex is central to understanding its transcriptional regulatory activity. Here, we generated an ORF66-null virus and confirmed that it fails to produce late genes and infectious virions. We show that ORF66 is incorporated into the vTA complex primarily through its interaction with ORF34, which is dependent upon a set of four conserved cysteinerich motifs in the C-terminal domain of ORF66. While both ORF24 and ORF66 occupy the canonical K8.1 late gene promoter, their promoter occupancy requires the presence of the other vTAs, suggesting that sequence-specific, stable binding requires assembly of the entire complex on the promoter. Additionally, we found that ORF24 expression is impaired in the absence of a stable vTA complex. This work extends our knowledge about the architecture of the KSHV viral preinitiation complex and suggests that it functions as a complex to recognize late gene promoters.
\end{abstract}

IMPORTANCE Kaposi's sarcoma-associated herpesvirus (KSHV; human herpesvirus 8) is an oncogenic gammaherpesvirus that is the causative agent of multiple human cancers. The release of infectious virions requires the production of capsid proteins and other late genes, whose production is transcriptionally controlled by a complex of six virally encoded proteins that hijack the host transcription machinery. It is poorly understood how this complex assembles or what function five of its six components play in transcription. Here, we demonstrate that ORF66 is an essential component of this complex in KSHV and that its inclusion in the complex depends upon its C-terminal domain, which contains highly conserved cysteine-rich motifs reminiscent of zinc finger motifs. Additionally, we examined the assembly of the viral preinitiation complex at late gene promoters and found that while sequence-specific binding of late gene promoters requires ORF24, it additionally requires a fully assembled viral preinitiation complex.

KEYWORDS Kaposi's sarcoma-associated herpesvirus, gammaherpesvirus, late gene, transcriptional activator

ammaherpesviruses, such as Kaposi's sarcoma-associated herpesvirus (KSHV) and Epstein-Barr virus (EBV), along with betaherpesviruses, such as human cytomegalovirus (HCMV), are double-stranded DNA viruses that co-opt and exploit the endoge-
Citation Didychuk AL, Castañeda AF, Kushnir LO, Huang CJ, Glaunsinger BA. 2020. Conserved $\mathrm{CX}_{\mathrm{n}} \mathrm{C}$ motifs in Kaposi's sarcoma-associated herpesvirus ORF66 are required for viral late gene expression and are essential for its interaction with ORF34. J Virol 94:e01299-19. https://doi.org/10.1128/JVI.01299-19.

Editor Rozanne M. Sandri-Goldin, University of California, Irvine

Copyright $\odot 2020$ American Society for Microbiology. All Rights Reserved.

Address correspondence to Britt A. Glaunsinger, glaunsinger@berkeley.edu. For a companion article on this topic, see https://doi.org/10.1128/JVI.01300-19.

Received 6 August 2019

Accepted 26 September 2019

Accepted manuscript posted online 2

October 2019

Published 6 January 2020 
nous cellular transcription machinery to facilitate viral gene expression. During the lytic phase of the life cycle, gammaherpesviral genes are expressed in a temporal cascade starting with immediate early genes, followed by early and then late genes. In all classes of viral genes, transcription depends upon host cellular RNA polymerase II (Pol II). Immediate early and early genes possess promoters similar to host promoters, with canonical TATA boxes. In contrast, late genes have minimal promoters, characterized by the presence of a noncanonical TATI box, which in KSHV is followed by an RVNYS motif (1-3).

Late genes in beta- and gammaherpesviruses are transcribed by a set of virally encoded genes, termed the viral transcriptional activators (vTAs), which form a complex (the vTA complex). The vTA complex, along with an unknown set of host general transcription factors, assemble on late gene promoters to form the viral preinitiation complex (vPIC) (4-6). Homologs of this complex are absent in alphaherpesviruses, which are thought to control late gene transcription by a distinct mechanism that depends on a canonical TATA box and Inr element (7). In KSHV, the vTAs are encoded by ORF18, ORF24, ORF30, ORF31, ORF34, and ORF66. Stop mutants of five of the six KSHV vTAs have been generated and tested, revealing that they share a phenotype in which late gene transcription fails to occur, ultimately preventing the release of infectious virions $(6,8-10)$. Disrupting protein-protein contacts within the complex also prevents late gene transcription, as preventing the incorporation of even the smallest vTA, ORF30 (77 amino acids [aa]), by disrupting its interaction with its binding partner, ORF18, completely prevents late gene transcription in both $\operatorname{KSHV}$ and $\operatorname{HCMV}(11,12)$. Although deletion of KSHV ORF66 has not been tested, an EBV mutant lacking the ORF66 homolog (BFRF2) fails to produce infectious virions due to a defect in late gene transcription (4). Thus, the six viral components of vPIC are absolutely required for late gene transcription.

It is well established that the six vTAs form a complex. One of these vTAs, ORF24, binds late gene promoter DNA and directly recruits Pol II to facilitate late gene expression (6). However, the function of the vTA complex as a whole beyond polymerase recruitment is unknown. ORF24 and its homologs are considered viral TATAbinding proteins (VTBP), as they have weak sequence similarity to host TBP and in silico modeling suggests that they may structurally mimic TBP (13). However, there is a dearth of structural or functional information for the remaining five vTAs.

Here, we confirm that KSHV ORF66 is essential for infectious virion production due to its role in late gene transcription. We demonstrate that ORF66 interacts with ORF18, ORF31, and ORF34 and that its interaction with ORF34, but not ORFs 18 and 31, occurs through the C-terminal domain of ORF66. Disruption of conserved cysteine-rich motifs within the C-terminal domain of ORF66 prevents late gene transcription due to disruption of the interaction between ORF66 and ORF34. We also demonstrate that the stable binding of ORF24 on late gene promoters requires both ORF66 and ORF30. These results extend our understanding of the architecture of the vTA complex as well as provide novel insights into its ability to recognize and bind late gene promoters in vivo.

(This article was submitted to an online preprint archive [14].)

\section{RESULTS}

ORF66 is essential for late gene expression in KSHV. KSHV ORF66 is a conserved protein with homologs in all beta- and gammaherpesviruses. Based on the phenotype observed upon deletion or mutation of the other KSHV vTAs $(6,8-10)$, we predicted that ORF66 would similarly be essential for viral replication and late gene expression in KSHV. We generated an ORF66-deficient recombinant KSHV bacterial artificial chromosome 16 (BAC16)-derived virus (ORF66.stop) using the Red recombinase system (15). As the coding region of ORF66 partially overlaps that of ORF67, we inserted two adjacent stop codons at amino acids 25 and 26 of ORF66 (Fig. 1A). We also engineered a corresponding mutant rescue ORF66 (ORF66.MR) to ensure that any phenotypes observed were not due to secondary mutations elsewhere in the bacterial artificial chromosome (BAC). The sequence of the recombinant BACs was confirmed by Sanger 
A

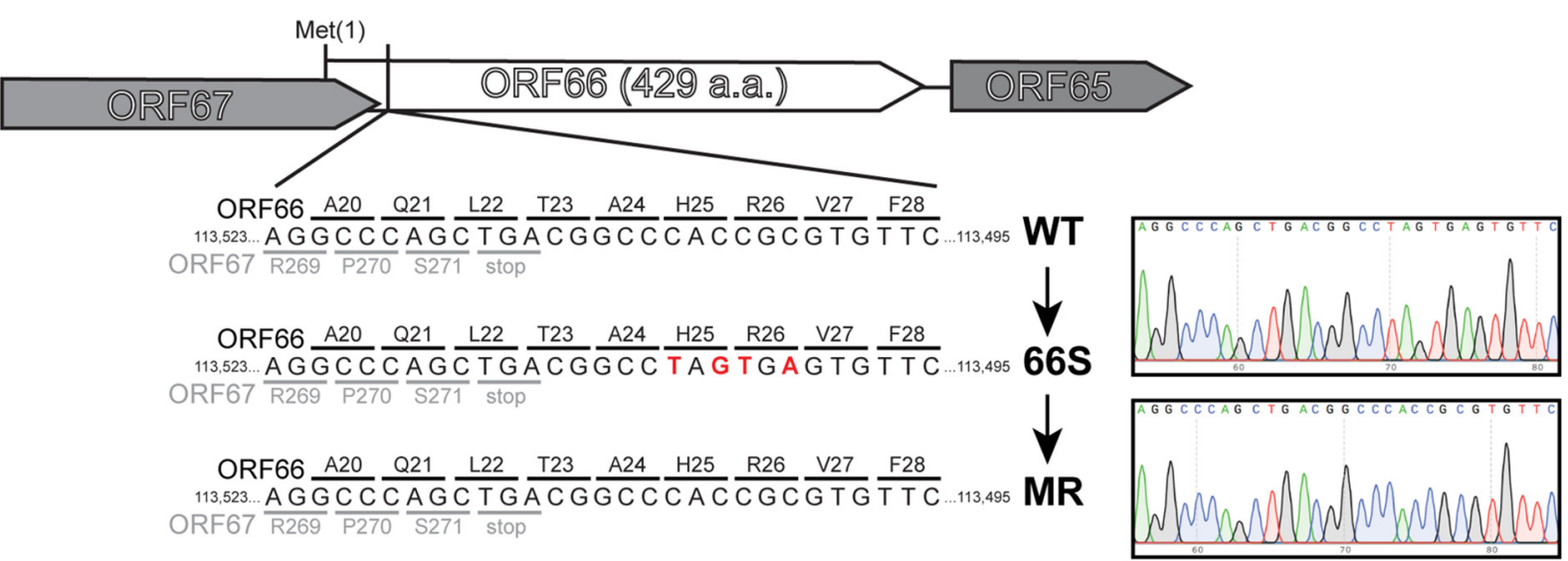

B

$326^{5}+2$

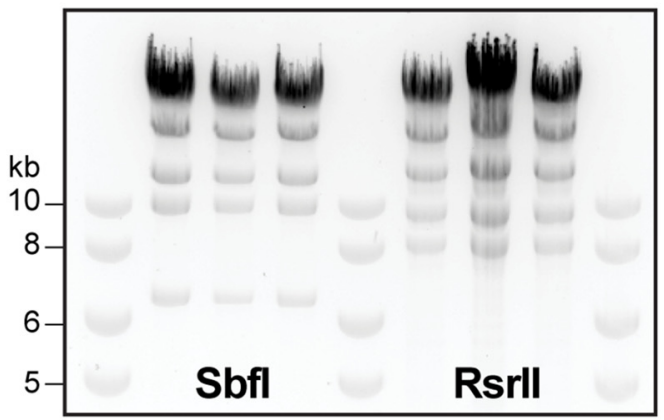

C Viral supernatant transfer

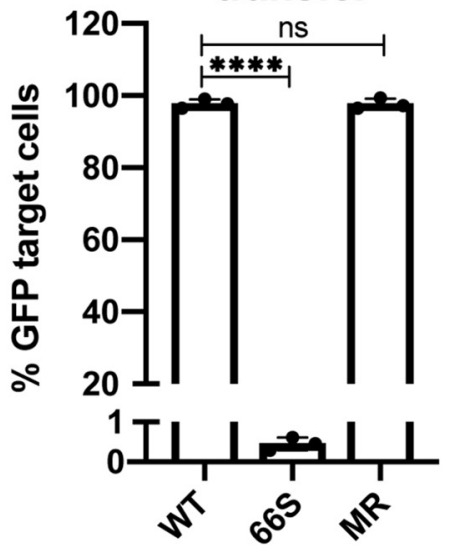

Viral DNA replication

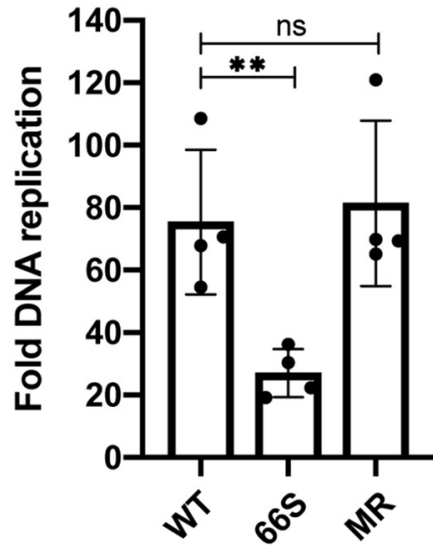

E

\section{WT} 66S

Reactivation:

MR
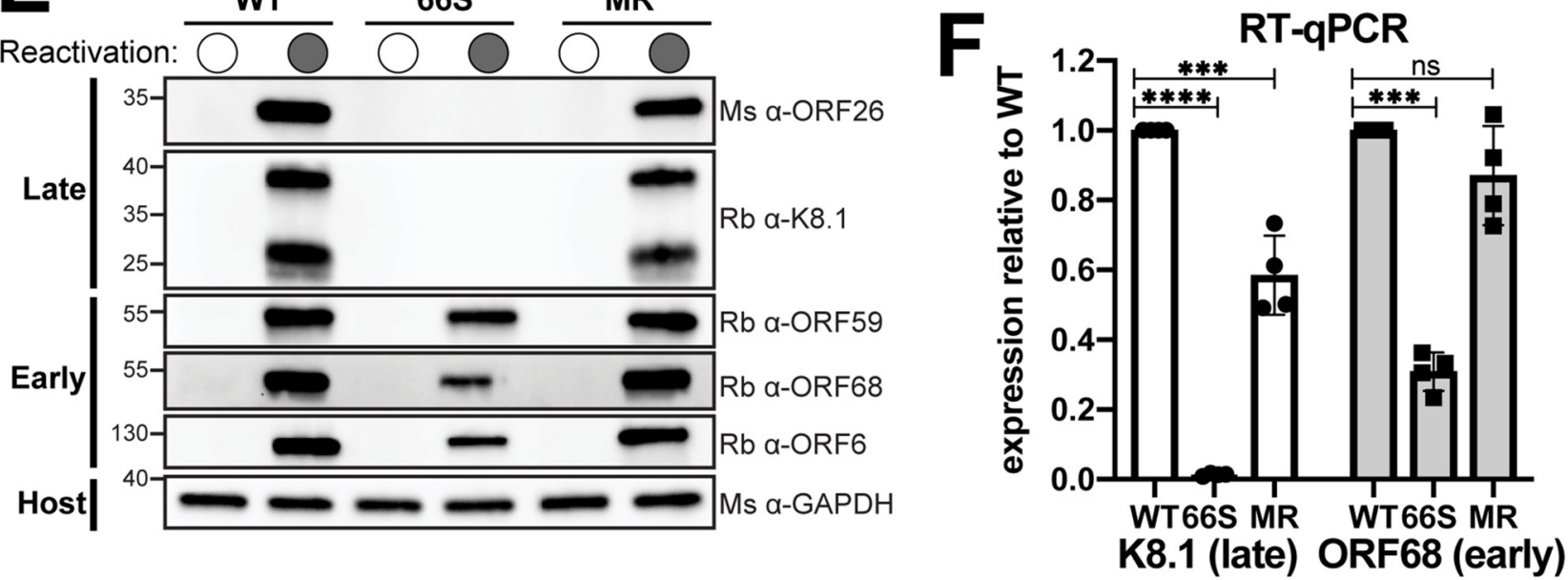

FIG 1 ORF66 is essential in KSHV and required for late gene transcription. (A) (Left) Diagram showing the genomic locus of ORF66 with surrounding genes ORF67 (which partially overlaps ORF66) and ORF65, depicting the location of introduced mutations. (Right) The mutations were confirmed by Sanger sequencing. (B) Digestion of the recombinant BACs with Sbfl or Rsrll demonstrates that the introduction of mutations did not introduce large-scale changes. (C) Infectious virion production was measured by supernatant transfer from reactivated iSLK cell lines followed by flow cytometry. Data are from three independent biological replicates, with statistics being calculated using an unpaired $t$ test. ****, $P<0.0001$; ns, not significant. GFP, green fluorescent protein. (D) Viral DNA replication was measured using qPCR before and after reactivation. Data are from four independent biological replicates, with statistics being calculated using an unpaired $t$ test. ${ }^{* *}, P<0.01$. (E) Western blotting of whole-cell lysate $(20 \mu \mathrm{g})$ reveals that early genes are largely unaffected by the ORF66.stop mutation but that late gene products cannot be detected. The numbers to the left of the blots are molecular weights (in kilodaltons); Rb, rabbit; Ms, mouse. (F) RT-qPCR reveals that the defect observed in the assay whose results are presented in panel $E$ are due to a transcriptional effect caused by the absence of ORF66. Data are from four independent biological replicates, with statistics being calculated using an unpaired $t$ test. ${ }^{* * *}, P<0.0001 ;{ }^{* *}, P<0.001$; ns, not significant. 
sequencing, and they were digested with Rsrll and Sbfl to ensure that no large-scale recombination had occurred during mutagenesis (Fig. $1 \mathrm{~A}$ and $\mathrm{B}$ ). We then generated latently infected inducible SLK (iSLK) cell lines harboring the KSHV genome on BAC16 (iSLK.BAC16 cells) by transfecting BACs into HEK293T cells, followed by coculture with iSLK cells maintained in medium with puromycin (iSLK-puro cells) harboring a doxycycline-inducible copy of ORF50 (RTA) to allow for efficient reactivation.

To assess whether ORF66 is essential for the completion of the viral life cycle, we monitored the production of infectious progeny virions using a supernatant transfer assay. The BAC16 system contains a constitutively expressed green fluorescent protein reporter gene, allowing for quantitation of infected target cells using flow cytometry. No detectable virus was produced by the ORF66.stop-infected cell line, whereas the wild-type (WT)- and ORF66.MR-infected cell lines produced sufficient virus to infect nearly all of the target cells (Fig. 1C). Thus, ORF66 is required for completion of the viral replication cycle.

We next tested whether ORF66 plays a role in replication of the viral genome. It is well established that late gene transcription is licensed by the initiation of viral genome replication (16-18). Although it has been previously reported that other vTA mutants do not exhibit a defect in viral genome replication $(6,8-10)$, we recently reported that mutations in ORF24 result in an $\sim 6$-fold defect in viral genome replication (1). Similarly, we found that the ORF66.stop virus had a modest $\sim 3$-fold defect in viral genome replication that was rescued in the ORF66.MR virus (Fig. 1D).

To further evaluate the role(s) of ORF66 in the viral replication cycle, we examined the expression of representative KSHV early and late genes in lytically reactivated iSLK cells containing WT, ORF66.stop, or ORF66.MR viruses by Western blotting (Fig. 1E) and reverse transcription (RT)-quantitative PCR (qPCR) (Fig. 1F). While the ORF66.stop infection produced the early proteins ORF59, ORF6, and ORF68, the late proteins K8.1 and ORF26 were not detectable (Fig. 1E). In contrast, both early and late proteins were expressed in the WT virus- and ORF66.MR-infected cells (Fig. 1E). RT-qPCR-based measurements of viral RNA from the ORF68 (early) and K8.1 (late) loci confirmed that the selective absence of late proteins in the ORF66.stop infections was due to a transcriptional defect (Fig. 1F). The moderate decrease in both the transcript and protein of early gene ORF68 is consistent with the observation that most viral transcripts are downregulated in the absence of functional ORF24 (1). We also observed less ORF6 (the single-stranded DNA binding protein involved in viral DNA replication) in the ORF66.stop-infected samples, which could explain the reduced levels of viral DNA replication observed in the ORF66.stop-infected cell line (Fig. 1D and E). Thus, while ORF66 modestly contributes to KSHV early gene expression and DNA replication, it is essential for late gene expression.

ORF66 is a component of the KSHV vTA complex. Based on our observation that ORF66 is essential for late gene transcription and our previous observation that ORF66 interacts with ORF18 (11), we sought to further characterize the interactions of ORF66 within the vTA complex (Fig. 2A). We began by assessing its association with the complex as a whole upon immunoprecipitation of different vTA components in transiently transfected HEK293T cells. We used FLAG magnetic beads to enrich for a FLAG-tagged ORF and tested whether the remaining five streptavidin (Strep)-tagged vTA complex components could be coimmunoprecipitated (Fig. 2B). As we previously showed, the VTA complex can be isolated by immunoprecipitation of ORF18 (Fig. 2B). We also tested if the complex could be isolated by immunoprecipitation of ORF31 or ORF66. Notably, although ORF31 was expressed as well as ORF18, it coimmunoprecipitated less ORF18 (and, thus, less ORF30) relative to immunoprecipitation by ORF18 or ORF66 (Fig. 2B). Overall, however, the structural integrity of the vTA complex is highlighted by the observation that immunoprecipitation of either ORF18, ORF31, or ORF66 resulted in the copurification of the remaining five vTA components.

To assess the contacts that ORF66 makes within the vTA complex, we tested its pairwise interactions with other components by coimmunoprecipitation (co-IP). As has 

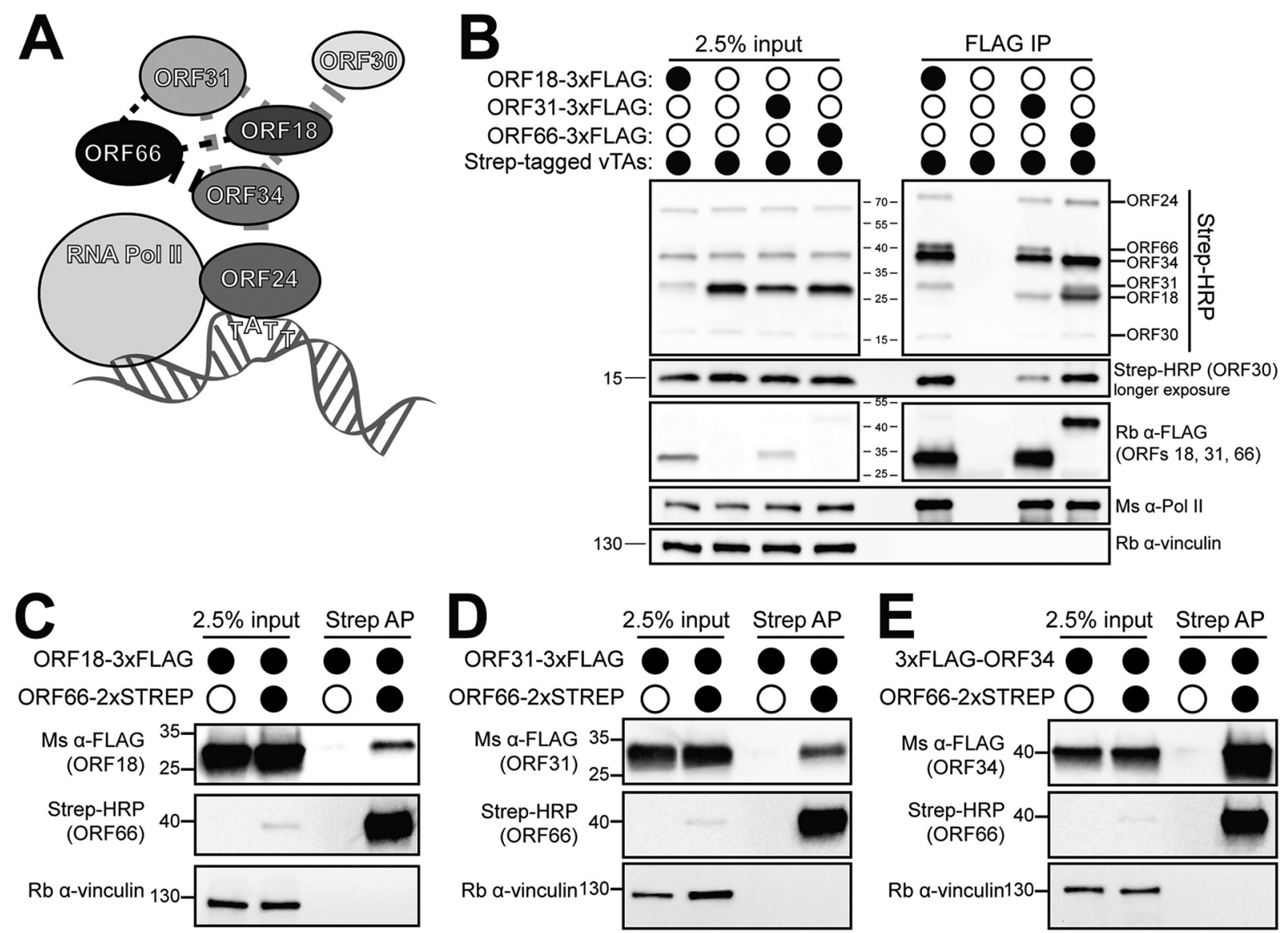

FIG 2 ORF66 is a component of the vTA complex and directly interacts with ORFs 18, 31, and 34. (A) Diagram of the vTA components in KSHV. Line weights indicate the relative strength of the interactions. (B) ORF18, ORF31, and ORF66 can immunoprecipitate the entire vTA complex. HEK293T cells were transiently transfected with FLAG- or Strep-tagged VTAs, and a co-IP was performed using anti-FLAG magnetic beads. (C to E) StrepTactinXT magnetic beads were used to isolate Strep-tagged ORF66 and demonstrate ORF18 (C), ORF31 (D), and ORF34 (E) co-IP with ORF66. The numbers to the left of the blots are molecular weights (in kilodaltons).

been previously observed, ORF66 interacts with ORF18 (Fig. 2C) (11), ORF31 (Fig. 2D), and, most robustly, ORF34 (Fig. 2E) (10). We were unable to detect direct proteinprotein interactions between ORF66 and ORF24 or ORF30 (data not shown). Thus, like ORF18 and ORF34, ORF66 exhibits multiple interactions within the vTA complex.

The C-terminal domain of ORF66 is essential for the interaction between ORF66 and ORF34. To define the region(s) of ORF66 responsible for mediating its VTA protein-protein interactions, we first created truncations of ORF66 that roughly divide the protein into two domains and then tested each for interaction with its binding partners by coimmunoprecipitation (Fig. 3A). Interestingly, both the $\mathrm{N}$ - and C-terminal domains of ORF66 could still interact to various degrees with ORF18 (Fig. 3B) and ORF31 (Fig. 3C). In contrast, the interaction with ORF34 mapped exclusively to the ORF66 C-terminal domain (Fig. 3D). The robust interaction between ORF34 and ORF66 (in comparison to the significantly weaker interactions between ORF66 and ORF18 or ORF31) suggests that this interaction may drive the incorporation of ORF66 into the vTA complex.

The C-terminal domain of ORF66 contains conserved $\mathrm{Cx}_{\mathrm{n}} \mathrm{C}$ motifs required for late gene expression. We next sought to further refine which residues underlie the robust interaction between the C-terminal domain of ORF66 and ORF34. We performed a multiple-sequence alignment of the C-terminal domain of KSHV ORF66 and its homologs in the gammaherpesviruses murine gammaherpesvirus 68 (mu66) and EBV (BFRF2), along with homologs from the betaherpesviruses murine cytomegalovirus (M49), HCMV (UL49), human herpesvirus 6A (U33), and human herpesvirus 7 (UL49) 


\section{A}

ORF66 1-429-2xStrep
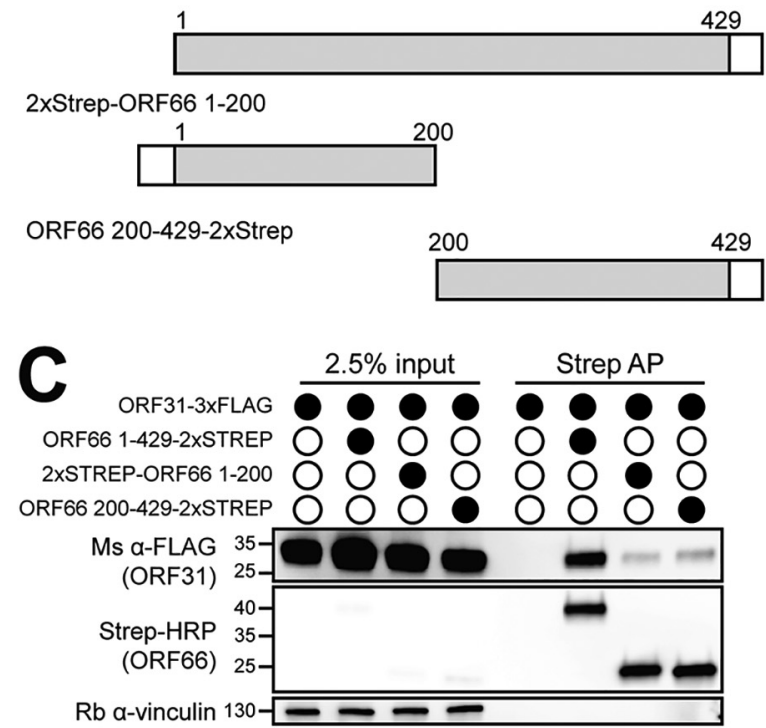
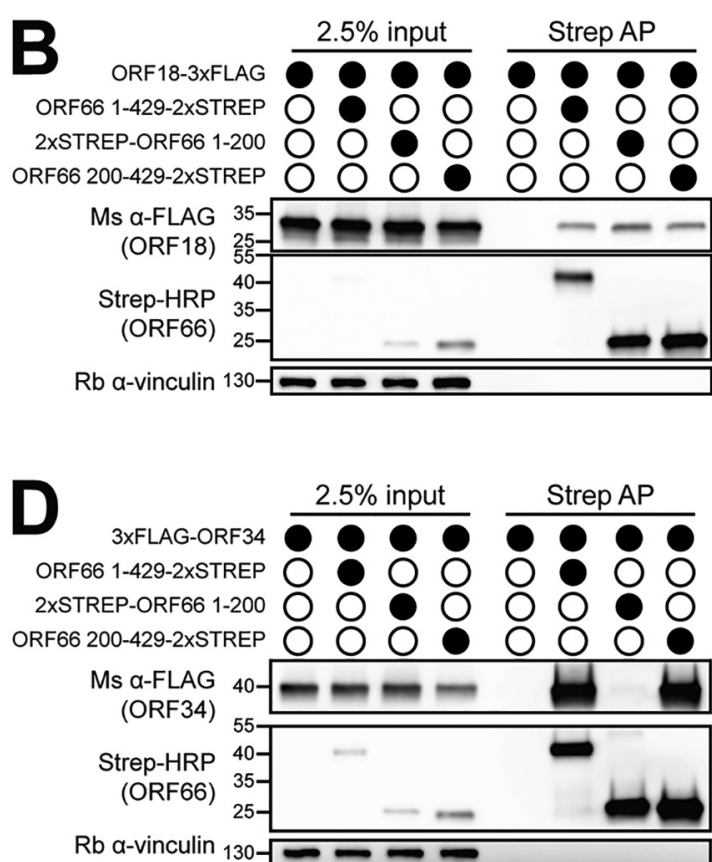

FIG 3 The C-terminal domain of ORF66 is essential for protein-protein interactions within the vTA complex. (A) Diagrams of the constructs used to test the domain structure of ORF66. (B to D) HEK293T cells were transiently transfected with Strep-tagged ORF66 and the indicated FLAG-tagged VTA and then coimmunoprecipitated with StrepTactinXT beads (Strep AP), followed by Western blotting. The numbers to the left of the blots are molecular weights (in kilodaltons).

using the T-Coffee method (19) (Fig. 4A). It was immediately striking that four $\mathrm{Cx}_{\mathrm{n}} \mathrm{C}$ motifs (motifs I to IV) were perfectly conserved in the aligned region in all available sequences of beta- and gammaherpesvirus homologs of ORF66.

We individually mutated to alanine the nine conserved cysteines within motifs I to IV, along with four nonconserved cysteine residues interspersed between the conserved motifs (Fig. 4A). Importantly, all of the ORF66 mutants were expressed comparably to the wild-type protein when transfected into HEK293T cells, suggesting that these residues and, hence, the $C x_{n} C$ motifs are not critical for protein stability (Fig. 4B).

To determine if the $C x_{n} C$ motifs are necessary for late gene transcription, we used a previously described reporter assay wherein the firefly luciferase gene is under the control of an early gene promoter (from ORF57) or a late gene promoter (K8.1) (11). In addition to the firefly luciferase reporter, the six vTAs (including wild-type or mutant ORF66) were transiently transfected into HEK293T cells along with a renilla luciferase reporter to control for transfection efficiency. Using this assay, we found that eight of the nine conserved cysteines are required for late gene transcription (Fig. 4C). Although cysteine 301 (C301) is perfectly conserved and is part of the extended CxxCxxC in motif $\mathrm{I}$, its mutation does not affect late gene transcription (Fig. 4C).

We then tested the role of these ORF66 cysteine residues in the context of KSHV infection using a modified version of the assay described above. The virus in the ORF66.stop-infected iSLK cell line was reactivated from latency and then transfected with the luciferase reporter plasmids along with a plasmid containing wild-type or mutant ORF66. In this assay, the remaining components of the vTA complex were supplied by the viral genome. The results in infected cells paralleled those obtained in HEK293T cells, as the mutants with the same eight conserved cysteine residue mutations in motifs I to IV (C295A, C298A, C341A, C344A, C393A, C399A, C424A, C427A) selectively failed to activate the luciferase late gene reporter (Fig. 4D). In contrast, the mutant with C301A in motif I and the four mutants with cysteine mutations interspersed between motifs I and IV activated the late promoter similarly to wild-type ORF66 (Fig. 4D). In conclusion, our results show that, with the exception of C301 at the 


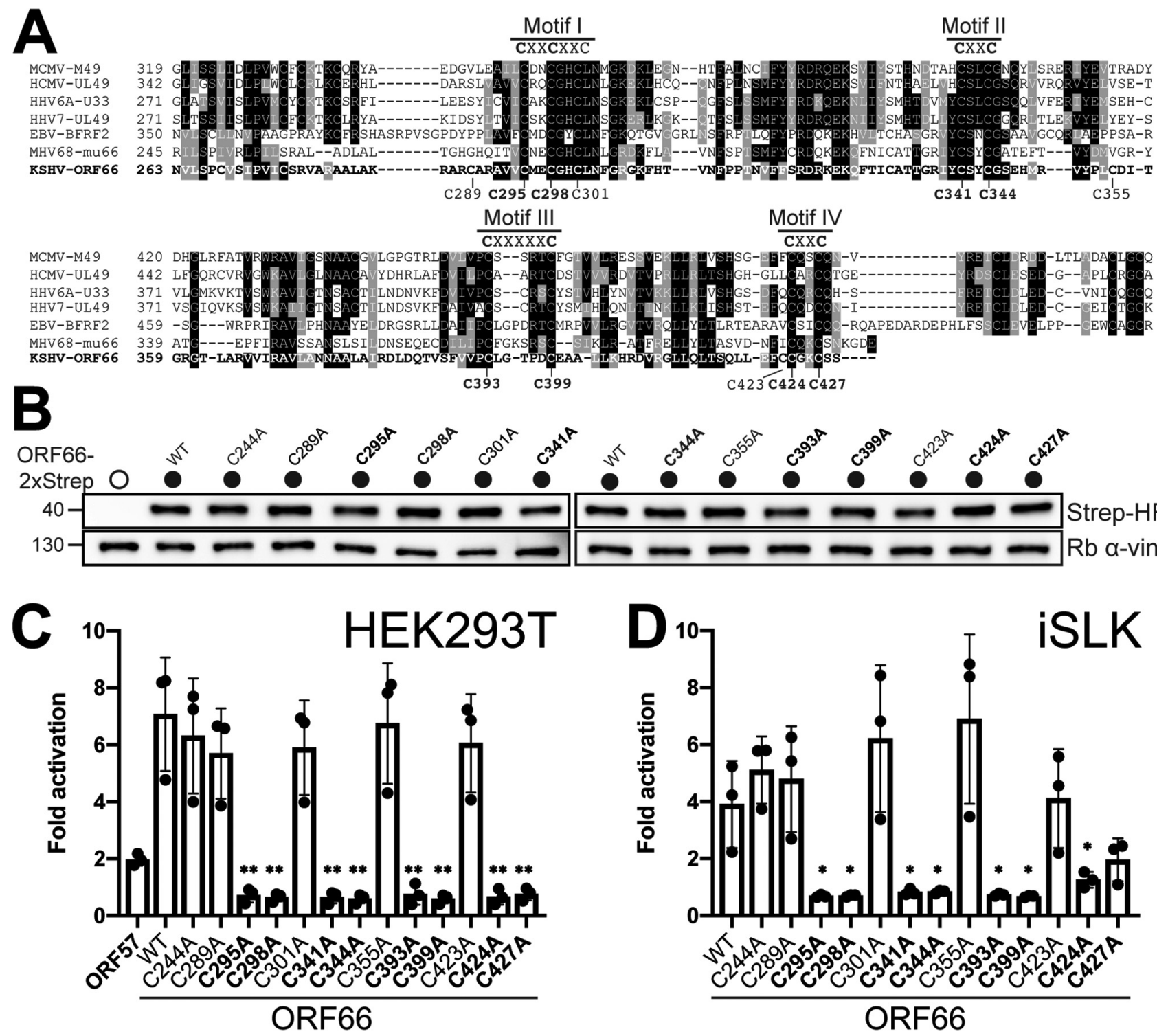

FIG 4 ORF66 contains conserved $\mathrm{Cx}_{\mathrm{n}} \mathrm{C}$ motifs essential for late gene transcription. (A) Multiple-sequence alignment between KSHV ORF66 and homologs from related beta- and gammaherpesviruses. The cysteine residues used in subsequent experiments are indicated below the alignment, and the presence of conserved $\mathrm{Cx}_{n} C$ motifs is indicated above the alignment. Residues conserved in $>50 \%$ of sequences are shaded, with identical residues in black and similar residues in gray. Bold indicates cysteines that affect late gene transcription. MCMV, murine cytomegalovirus, HHV6A, human herpesvirus 6A; HHV7, human herpesvirus 7; MHV68, murine gammaherpesvirus 68. (B) Western blot of whole-cell lysate (15 $\mu \mathrm{g})$ from HEK293T cells transiently transfected with plasmids containing wild-type or mutant Strep-tagged ORF66. The numbers to the left of the blots are molecular weights (in kilodaltons). (C) HEK293T cells were transfected with plasmids encoding the six vTAs (including either wild-type or mutant ORF66), the pGL4.16 firefly luciferase plasmid under the control of either the K8.1 or ORF57 promoter, and the pRL-TK renilla luciferase plasmid as a transfection control. After $24 \mathrm{~h}$, the cell lysates were harvested and luciferase activity was measured. (D) iSLK cells were transfected with plasmids encoding wild-type or mutant ORF66, the pGL4.16 luciferase plasmid under the control of the K8.1 promoter, and the pRL-TK renilla luciferase plasmid control. In both panels $C$ and $D$, fold activation was normalized to that for a control in which an empty vector replaced the vTAs (C) or ORF66 (D). Data are from three independent biological replicates, with statistics being calculated using an unpaired $t$ test. ${ }^{* *}, P<0.01$; ${ }^{*}, P<0.05$.

end of motif $\mathrm{I}$, the conserved cysteines comprising the four $\mathrm{Cx}_{\mathrm{n}} \mathrm{C}$ motifs in the C-terminal domain of ORF66 are critical for late gene transcription.

The $\mathbf{C x}_{\mathbf{n}} \mathbf{C}$ motifs in the C-terminal domain of ORF66 are required for interaction with ORF34 and its incorporation into the vPIC. We next evaluated what role the $\mathrm{Cx}_{\mathrm{n}} \mathrm{C}$ motifs in ORF66 might play in the assembly of the vTA complex. We selected representative mutants with cysteine mutations from the four motifs (C295, C341, C393, C424) and tested their ability to interact with each of the binding partners of ORF66 (Fig. 5A to C). All of the mutants retained wild-type levels of binding to ORF18 or ORF31 

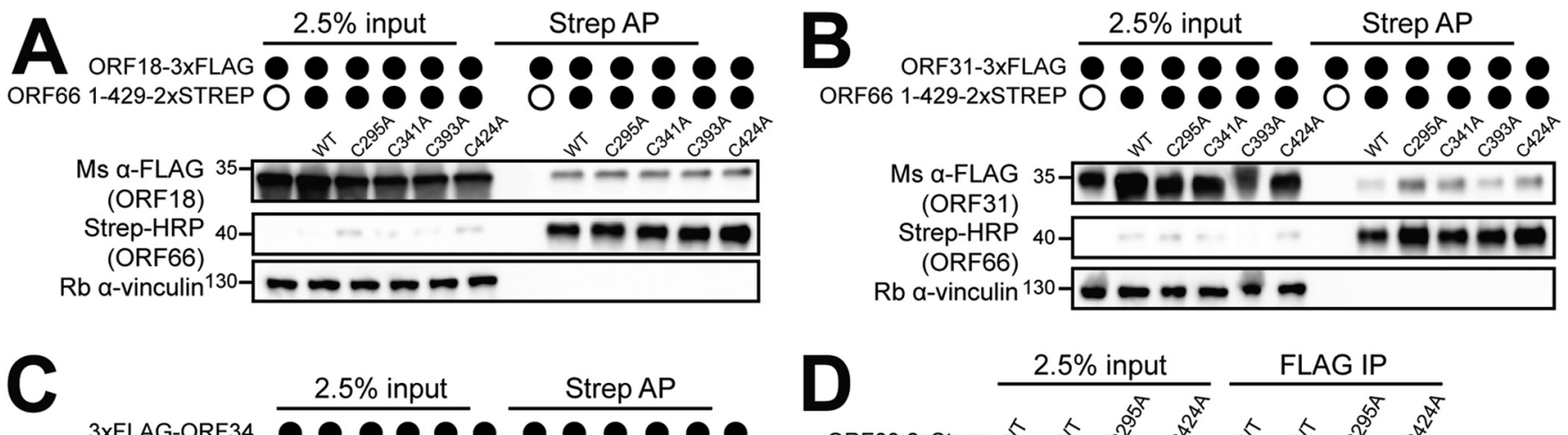

3xFLAG-ORF34

$2.5 \%$ input Strep AP ORF66 1-429-2xSTREP
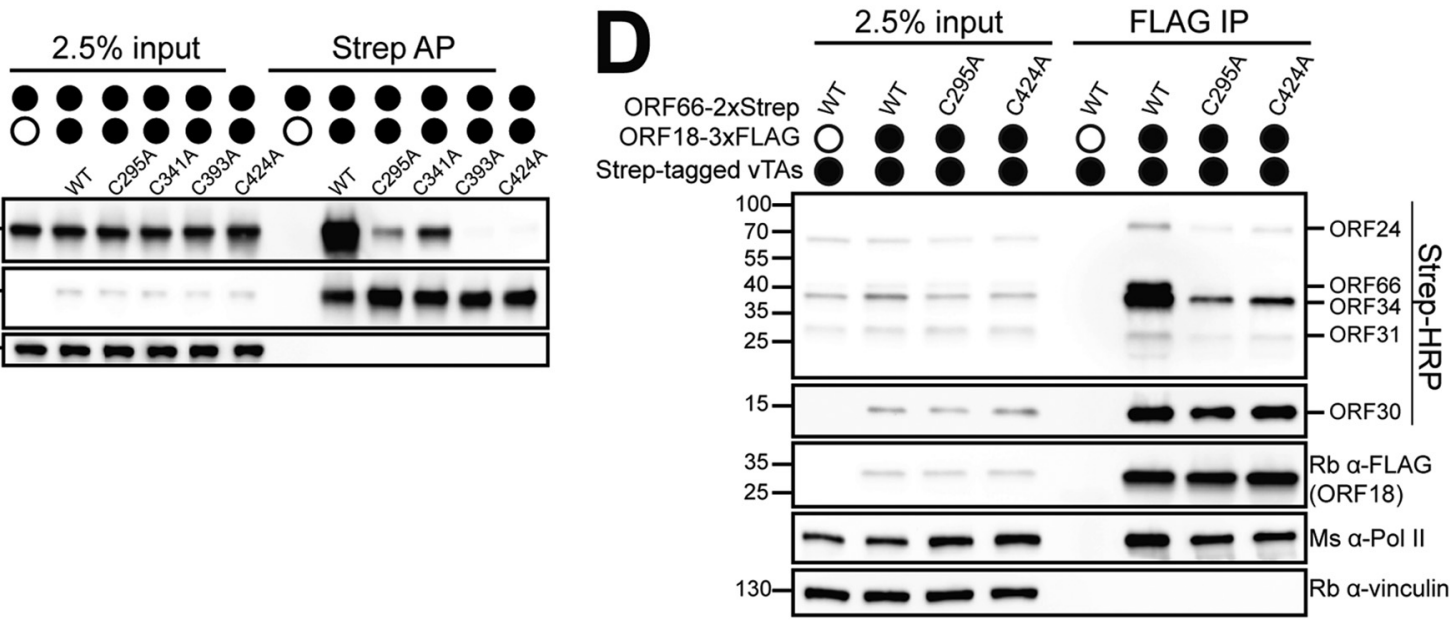

FIG 5 The $\mathrm{CX}_{n} \mathrm{C}$ motifs in ORF66 are required for interaction with ORF34. (A to C) HEK293T cells were transiently transfected with wild-type or mutant Strep-tagged ORF66 and the indicated FLAG-tagged vTA and then coimmunoprecipitated with StrepTactinXT beads, followed by Western blotting. (D) HEK293T cells were transiently transfected with wild-type or mutant Strep-tagged ORF66 along with FLAG-tagged ORF18, ORF24, ORF30, ORF31, and ORF34. A co-IP with anti-FLAG magnetic beads was performed, followed by Western blotting. The numbers to the left of the blots are molecular weights (in kilodaltons).

(Fig. 5A and B). In contrast, the mutants with mutations in motif I (C295A) and motif II (C341A) displayed greatly reduced binding to ORF34, and the mutants with mutations in motif III (C393A) and motif IV (C424A) failed to interact with ORF34 (Fig. 5C). This is consistent with our previous observation that the C-terminal domain of ORF66 is essential for its interaction with ORF34.

We then tested if ORF66 mutants with significantly weakened ORF34 binding (i.e., the motif I C295A mutant) or fully impaired ORF34 binding (i.e., the motif IV C424A mutant) impacted the incorporation of ORF66 into the vTA complex (Fig. 5D). We isolated the VTA complex by immunoprecipitation of FLAG-tagged ORF18 in the presence of the remaining 5 Strep-tagged VTA proteins, including wild-type or mutant ORF66. Neither the ORF66 C295A mutant nor the ORF66 C424A mutant was incorporated into the VTA complex (Fig. 5D), despite the fact that these mutants retained pairwise interactions with ORF18 and ORF31 (Fig. 2). Notably, the remaining vTA components (ORFs 18, 24, 30,31, and 34) still assembled into the complex in the absence of ORF66. Thus, the robust ORF66 $\mathrm{Cx}_{n} \mathrm{C}$ motif-dependent interaction with ORF34, but not the weaker interactions of ORF66 with ORF18 and ORF31, is essential to recruit ORF66 into the vTA complex.

An intact vPIC is required for stable binding of ORF66 and ORF24 at late gene promoters in vivo. ORF24 is the only VTA known to directly contact promoter DNA, although other vTAs likely colocalize at late gene promoters via protein-protein interactions within the complex $(1,6)$. However, given that ORF66 possesses $C x_{n} C$ motifs, which are frequently found in nucleic acid binding proteins (20), we considered that it might independently bind promoter DNA. We also sought to test whether other VTAs, including ORF66, contribute to ORF24 promoter specificity or binding during infection.

In this regard, we generated KSHV containing C-terminally hemagglutinin (HA)tagged ORF66 in an otherwise WT BAC16 background (66HA) or in BAC16 lacking 
ORF24 $(66 \mathrm{HA} / 24 \mathrm{~S})$ to evaluate whether ORF66 associates with the late gene promoter and, if so, whether its association requires ORF24. We also generated KSHV that contained an N-terminally HA-tagged ORF24 (HA24) (1) and that lacked either ORF66 (HA24/66S) or ORF30 (HA24/30S) to determine whether promoter binding by ORF24 is influenced by other vTAs. Unlike ORF66, the ORF30 vTA is a small protein with no predicted nucleic acid binding properties, and its only connection with the complex occurs through ORF18 (Fig. 2A) (11). Thus, ORF30 deletion should enable evaluation of the general importance of the vTA complex in ORF24 late gene promoter binding. We generated the recombinant BACs and iSLK cell lines as described above and digested the BACs with Rsrll and Sbfll to ensure that no large-scale recombination occurred during mutagenesis (Fig. 6A).

We first characterized each of the infected cell lines for their ability to produce infectious virions and express early and late genes upon lytic reactivation. As expected, iSLK cells containing HA24 KSHV and 66HA KSHV produced infectious virus, as measured using a supernatant transfer assay, although in the case of 66HA KSHV the levels were modestly reduced (by $\sim 40 \%$ ) relative to those for WT KSHV (Fig. 6B). This reduction could be caused by an effect of the tag on ORF66 stability or by the location of the epitope tag in the viral genome, as it changes the $5^{\prime}$ untranslated region of the neighboring gene for ORF65. As expected, KSHV mutants lacking any one of the vTAs (HA24/30S, HA24/66S, or 66HA/24S) produced no detectable infectious virions (Fig. 6B).

Western blotting confirmed that all of the engineered viruses expressed the representative early proteins ORF59, ORF68, and ORF6 (Fig. 6C). However, the late proteins ORF26 and K8.1 were not produced in cell lines lacking any of the vTAs (Fig. 6C). Late proteins were produced in the 66HA KSHV-infected iSLK cells, although in agreement with the virion production data, their levels were slightly reduced relative to those produced in cells infected with KSHV containing untagged ORF66 (WT) (Fig. 6C). Finally, we confirmed expression of the HA-tagged ORF24 and ORF66 by immunoprecipitation with anti-HA beads (Fig. 6D and E), which was necessary as both proteins are present at a low abundance and cannot be easily detected in whole-cell lysates. Interestingly, the levels of HA-ORF24 (but not ORF66-HA) were reduced upon deletion of either ORF30 or ORF66, suggesting that ORF24 expression is bolstered by an intact vTA complex (Fig. 6D).

We next performed chromatin immunoprecipitation (ChIP) using the HA tag on endogenous ORF24 or ORF66 and quantified the amount of associated DNA by qPCR. As anticipated, both HA-ORF24 and ORF66-HA bound to the promoter of the K8.1 late gene but not to that of the ORF37 early gene (Fig. 6F). Notably, ORF66-HA binding to the K8.1 promoter did not occur in the absence of ORF24 (66HA/24S KSHV), suggesting that it does not independently bind the late gene promoter (Fig. 6F). Surprisingly, we detected no HA-ORF24 binding at the K8.1 promoter during infection with viruses lacking either ORF66 or ORF30 (Fig. 6F). It is possible that the ChIP assay is not sensitive enough to detect DNA associated with the reduced levels of HA-ORF24 in these cells (Fig. 6D). However, we favor the hypothesis that although ORF24 alone displays sequence-specific DNA binding in vitro (6), its stable association with late promoters in cells may require an intact vTA complex. In summary, our results suggest that the sequence-specific binding of ORF24 at late gene promoters is bolstered by the vTA complex.

\section{DISCUSSION}

Here, we demonstrate that KSHV ORF66, like the other 5 vTAs $(6,8-10)$, is necessary for completion of the lytic replication cycle due to its critical role in late gene transcription. Additionally, our results complement previous work (11) in demonstrating that disruption of any of the protein-protein interactions within the complex, even when the remaining contacts between other vTA complex components are maintained, prevents late gene transcription. Despite recent progress in understanding the overall organization of the vTA complex, the role of each vTA is poorly understood. We mapped the interactions between ORF6 6 and other members of the vTA complex and 

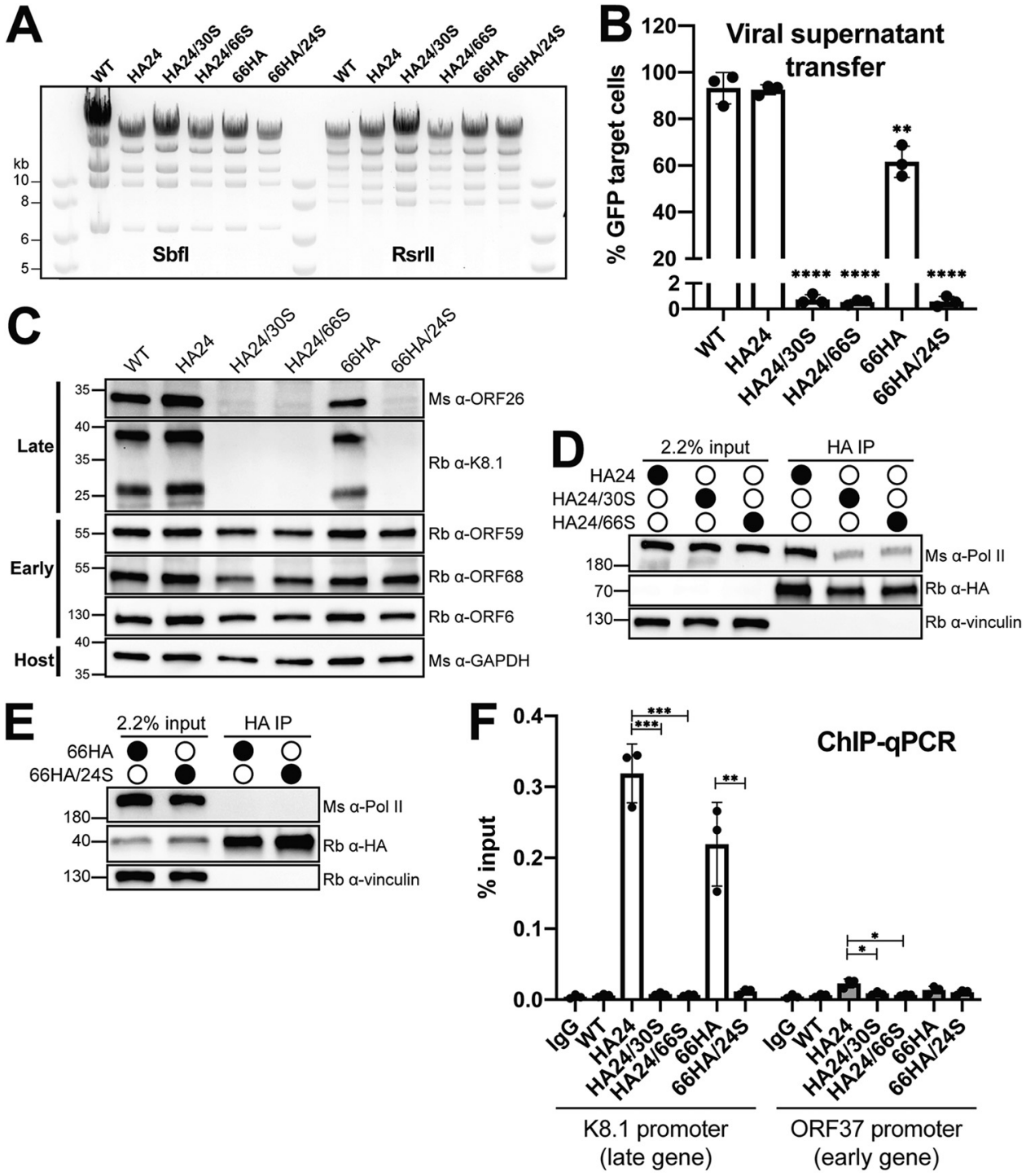

FIG 6 ORF24 does not bind to late gene promoters in the absence of ORF30 or ORF66. iSLK cell lines were created using the recombinant BAC16 system. HA tags were added to the endogenous copies of the N terminus of ORF24 (HA24) or the $\mathrm{C}$ terminus of ORF66 (66HA). In select BACs, ORF24, ORF30, or ORF66 was deleted by the introduction of a stop codon early in the $\operatorname{ORF}(24 \mathrm{~S}, 30 \mathrm{~S}$, and $66 \mathrm{~S}$ respectively). (A) Digestion of the recombinant BACs with Sbfl or Rsrll demonstrates that recombination did not introduce large-scale changes. (B) Infectious virion production was measured by supernatant transfer from reactivated iSLK cell lines followed by flow cytometry. Data are from three independent biological replicates, with statistics being calculated using an unpaired $t$ test. ${ }^{* * * *}, P<0.0001$; **, $P<0.01$. (C) Western blot of whole-cell lysate $(25 \mu \mathrm{g})$ from iSLK cell lines showing the relative levels of representative early and late genes. (D) Western blots showing the expression of HA-ORF24 in different cell lines. Proteins were immunoprecipitated with HA beads to enrich for ORF24, which is present at a low abundance. (E) Western blots showing the expression of ORF66-HA in different cell lines. Proteins were immunoprecipitated with HA beads to enrich for ORF66, which is present at a low abundance. (F) ChIP-qPCR from the indicated cell lines was performed using an anti-HA antibody. The associated DNA from either the K8.1 promoter (a late gene promoter) or the ORF37 promoter (an early gene promoter) was quantified using promoter-specific primers. Data are from three independent biological replicates, with statistics being calculated using an unpaired $t$ test. ${ }^{* *}, P<0.001 ;{ }^{* *}$, $P<0.01 ;{ }^{*}, P<0.05$. The numbers to the left of the blots are molecular weights (in kilodaltons).

identified four conserved $\mathrm{Cx}_{\mathrm{n}} \mathrm{C}$ motifs within the C-terminal domain of ORF66. When mutated, these motifs abolish late gene transcription due to a drastic reduction in the ability of ORF66 to bind ORF34. We show that ORF66 is present at late gene promoters during infection but does not bind in the absence of ORF24. ORF24 binding at late gene promoters requires both ORF66 and ORF30, suggesting that the presence of all six VTAs at late gene promoters is necessary for stable binding of the vPIC.

The vTA complex displays remarkable physical and functional interconnectivity. 
While ORF34 was originally proposed to be the scaffold upon which the other vTAs assemble, it is clear that both ORF66 and ORF18 directly engage in interactions with three and four vTAs, respectively, suggesting that the complex is stabilized by numerous protein-protein interactions between multiple core components. Furthermore, all six members of the VTA complex must be present and individual protein-protein interactions must be maintained in order for late gene transcription to occur. Disruption of the interaction between ORF6 6 and ORF34 or the interaction between ORF30 and ORF18 (11) not only changes the composition of the vTA complex (by preventing the inclusion of ORF66 or ORF30, respectively) but also destabilizes the complex, even though multiple contacts between other vTAs exist. Ultimately, determining the individual structures of vTAs and the architecture of the complex at a molecular resolution will be key to interpreting the roles of known protein-protein contacts and deciphering their functions in late gene transcription.

Three of the six vTAs-ORF31, ORF34, and ORF66 - contain conserved cysteine/ histidine motifs that, in the context of zinc finger domains, are frequently found in nucleic acid binding proteins. However, the observation that ORF66 associates only with the K8.1 promoter in the presence of ORF24 suggests that it does not bind promoter DNA (at least not alone) and is more likely to be recruited indirectly through contacts with the vTA complex. Thus, although the $\mathrm{Cx}_{\mathrm{n}} \mathrm{C}$ motifs in ORF66 are required for late gene expression, they appear to be essential for an interaction with ORF34 rather than to contribute to promoter-specific binding by the vPIC. Mutations within the $\mathrm{Cx}_{\mathrm{n}} \mathrm{C}$ motifs did not change the expression or stability of ORF66 in cells or its interactions with ORF18 or ORF31, making it unlikely that these mutations result in global unfolding. Instead, we hypothesize that they serve to structurally stabilize a region of the protein that directly interacts with ORF34.

Although ORF66 expression was not dependent on the other vTAs, we found that ORF24 protein levels decreased in the absence of ORF66 or ORF30, consistent with an observed downregulation of the majority of KSHV genes in the absence of a functional vTA complex $(1,9)$. Uncovering the links between ORF24 expression and the vTA complex could lead to new insights regarding the regulation of late gene transcription. For example, the vTA complex or one of its components could be responsible for stabilization of the low-abundance ORF24 protein. Alternatively, a component(s) of the vTA complex could be important for the correct localization of ORF24 to the nucleus, as has been observed for the KSHV DNA replication complex (21). Regardless of the mechanism involved, it is important to keep in mind that a reduction in ORF24 levels might contribute to the defects observed in cell lines where the other vTAs are deleted (6, 8-10).

ORF24 has weak sequence similarity but predicted structural similarity to the host TATA-binding protein (TBP) (13). Indeed, ORF24 has the ability to bind DNA directly (6) and is present at late gene promoters in vivo (1). However, there are clear functional differences between ORF24 and TBP. ORF24 binds a TATT-containing probe in vitro yet does not bind the probe when the TATT motif is mutated to CCCC (6). Its homolog from EBV, BCRF1, binds both TATT- and TATA-containing motifs in vitro but does not bind an unrelated probe (22). These observations suggest that ORF24 has some inherent sequence specificity for T/A-rich elements, similar to the findings for TBP. However, many promoters that lack canonical TATA boxes are bound by TBP in vivo (23), in contrast to the specificity for TATT(T/A)AAA plus RVNYS promoters that are bound by the VPIC (1). Thus, the mechanism by which ORF24 specifically occupies the minimalistic late gene promoters remains a central unanswered question in viral late gene biology. We detected no binding of ORF24 at late gene promoters of viruses lacking either ORF30 or ORF66. These results suggest that despite the apparent sequence specificity of ORF24 in vitro (6), sequence-specific binding during infection requires the other components of the VTA complex. Neither ORF66 nor ORF30 directly interacts with ORF24, and thus, the regulation and stabilization of ORF24 binding are presumably orchestrated through the web of protein-protein interactions within the vTA complex.

Despite significant progress in defining the components of the vTA complex and the 
protein-protein interactions in which they participate, the functional contribution of each VTA within the VPIC remains largely enigmatic. By analogy to the similarity between ORF24 and TBP, the remaining five vTAs may be mimicking other core host transcription factors. Alternatively, the vTAs may be responsible for the recruitment of host transcription factors. Either of these possibilities allows for a contribution to sequence-specific binding by the non-ORF24 vTAs, although such binding may be observed only in the context of the fully assembled late gene vPIC. Determining which host transcription factors are present at late gene promoters is of utmost importance in deciphering the mechanism by which the vTAs operate.

\section{MATERIALS AND METHODS}

Plasmids. All plasmids described below were generated using InFusion cloning (Clontech), unless indicated otherwise; all have been deposited in Addgene. ORF66 was subcloned into the BamHI and Xhol sites of pcDNA4/TO-2xStrep (C-terminal tag) to generate pCDNA4/TO-ORF66-2xStrep (Addgene plasmid 130953). ORF66 aa 1 to 200 were cloned into the Notl and Xhol sites of pcDNA4/TO-2xStrep (N-terminal tag) to generate pcDNA4/TO-2xStrep-ORF66 1-200 (Addgene plasmid 130954) and ORF66 aa 200 to 429 were cloned into the BamHI and Xhol sites of pcDNA4/TO-2xStrep (C-terminal tag) to generate pcDNA4/ TO-ORF66 200-429-2xStrep (Addgene plasmid 130955). Point mutations in pcDNA4/TO-ORF66-2xStrep (Addgene plasmids 131109 to 131121) were generated using inverse PCR site-directed mutagenesis with Phusion DNA polymerase (New England Biolabs) with the primers listed in Table 1. PCR products from inverse PCR were Dpnl treated, ligated using T4 polynucleotide kinase and T4 DNA ligase, and transformed into Escherichia coli XL-1 Blue cells. ORF66-2xStrep, ORF30-2xStrep, and ORF24-3xFLAG were subcloned into the Agel and EcoRI sites of pLJM1 that had been modified to confer zeocin resistance (Addgene plasmids 130957 to 130959). Plasmid K8.1 Pr pGL4.16 (Addgene plasmid 120377) contains the minimal K8.1 promoter, and ORF57 Pr pGL4.16 (Addgene plasmid 120378) contains a minimal ORF57 early gene promoter; these plasmids have been described previously (11). Plasmid K8.1 Pr pGL4.16+Ori (Addgene plasmid 131038) contains the left origin of replication along with a 100-bp fragment of the K8.1 promoter and has been described previously (1). Plasmids pCDNA4/TO-ORF18-2xStrep (Addgene plasmid 120372), pcDNA4/TO-ORF24-2xStrep (Addgene plasmid 129742), pcDNA4/TO-ORF30-2xStrep (Addgene plasmid 129743), pcDNA4/TO-ORF31-2xStrep (Addgene plasmid 129744), and pcDNA4/TO2xStrep-ORF34 (Addgene plasmid 120376) have been described previously (11). Plasmid pRL-TK (Promega) was kindly provided by Russell Vance. Lentiviral packaging plasmids psPAX2 (Addgene plasmid 12260) and pMD2.G (Addgene plasmid 12259) were gifts from Didier Trono.

Cell lines. HEK293T cells (ATCC CRL-3216) were maintained in Dulbecco modified Eagle medium (DMEM) supplemented with 10\% fetal bovine serum (FBS) (Seradigm). HEK293T cells constitutively expressing ORF662xStrep (HEK293T-ORF66), ORF24-3xFLAG (HEK293T-ORF24), ORF30-2xStrep (HEK293T-ORF30), or 2xStrepORF34 (HEK293T-ORF34) were maintained in DMEM supplemented with $10 \% \mathrm{FBS}$ and $500 \mu \mathrm{g} / \mathrm{ml}$ zeocin.

iSLK-puro cells were maintained in DMEM supplemented with $10 \% \mathrm{FBS}$ and $1 \mu \mathrm{g} / \mathrm{ml}$ puromycin. The iSLK cell line harboring the KSHV genome on the bacterial artificial chromosome BAC16 and a doxycycline-inducible copy of the KSHV lytic transactivator RTA (iSLK-BAC16 cells) has been previously described (15). All iSLK-BAC16 cell lines were maintained in DMEM supplemented with 10\% FBS, $1 \mathrm{mg} / \mathrm{ml}$ hygromycin, and $1 \mu \mathrm{g} / \mathrm{ml}$ puromycin (iSLK-BAC16 medium).

Cell line establishment and viral mutagenesis. HEK293T cells stably expressing ORF66, ORF24, and ORF30 were generated by lentiviral transduction for the purpose of propagating KSHV deletion mutants lacking these essential genes. Lentivirus was generated in HEK293T cells by cotransfection of pLJM1ORF66, -ORF24, or -ORF30 along with the packaging plasmids pMD2.G and psPAX2. After $48 \mathrm{~h}$, the supernatant was harvested and syringe filtered through a $0.45-\mu \mathrm{m}$-pore-size filter (Millipore). The supernatant was diluted 1:2 with DMEM, and Polybrene was added to a final concentration of $8 \mu \mathrm{g} / \mathrm{ml}$. Freshly trypsinized HEK293T cells $\left(1 \times 10^{6}\right)$ were spinoculated in a 6 -well plate for $2 \mathrm{~h}$ at $1,000 \times \mathrm{g}$. After $24 \mathrm{~h}$, the cells were expanded to a 10-cm tissue culture plate and selected for 2 weeks in media supplemented with $500 \mu \mathrm{g} / \mathrm{ml}$ zeocin (Sigma).

All viral ORF mutants were generated using the scarless Red recombination system in BAC16 GS1783 Escherichia coli as previously described (15). The modified BACs were purified using a Nucleobond BAC 100 kit (Clontech). BAC quality was assessed by digestion with Rsrll and Sbfl (New England Biolabs). Latently infected iSLK cell lines with modified virus were generated by transfection of HEK293T cells (either WT cells or cells stably expressing the relevant essential viral ORF) with $5 \mu \mathrm{g}$ BAC DNA using the Polyjet reagent (SignaGen). On the following day, the transfected HEK293T cells were trypsinized and mixed 1:1 with freshly trypsinized iSLK-puro cells and treated with $30 \mathrm{nM}$ 12-O-tetradecanoylphorbyl13-acetate (TPA) and $300 \mathrm{mM}$ sodium butyrate for 4 days to induce lytic replication. iSLK cells were then selected in medium containing $300 \mu \mathrm{g} / \mathrm{ml}$ hygromycin B, $1 \mu \mathrm{g} / \mathrm{ml}$ puromycin, and $250 \mu \mathrm{g} / \mathrm{ml} \mathrm{G} 418$. The hygromycin B concentration was increased to $500 \mu \mathrm{g} / \mathrm{ml}$ and $1 \mathrm{mg} / \mathrm{ml}$ until all HEK293T cells died.

Virus characterization. For reactivation studies, $1 \times 10^{6}$ iSLK cells were plated in $10-\mathrm{cm}$ dishes for $16 \mathrm{~h}$ and then induced with $1 \mu \mathrm{g} / \mathrm{ml}$ doxycycline and $1 \mathrm{mM}$ sodium butyrate for an additional $72 \mathrm{~h}$. To determine the fold DNA induction in reactivated cells, the cells were scraped and triturated in the induced medium, and $200 \mu \mathrm{l}$ of the cell-supernatant suspension was treated overnight with $80 \mu \mathrm{g} / \mathrm{ml}$ proteinase $\mathrm{K}$ (Promega) in $1 \times$ proteinase $\mathrm{K}$ digestion buffer $(10 \mathrm{mM}$ Tris- $\mathrm{HCl}, \mathrm{pH} 7.4,100 \mathrm{mM} \mathrm{NaCl}, 1 \mathrm{mM}$ EDTA, $0.5 \%$ SDS), after which the DNA was extracted using a Quick-DNA Miniprep kit (Zymo). Viral DNA fold induction was quantified by qPCR using iTaq Universal SYBR green Supermix (Bio-Rad) on a 
TABLE 1 Primers used in this study

\begin{tabular}{|c|c|c|c|}
\hline Primer & Name & Sequence $\left(5^{\prime}-3^{\prime}\right)$ & Purpose \\
\hline 1 & ORF66-FL-F & TACCGAGCTCGGATCATGGCCCTGGATCAGCGC & Cloning \\
\hline 2 & ORF66-FL-R & CACCGCCTCCCTCGAGGGAGGAACACTTCCCGCAAC & Cloning \\
\hline 3 & 66-LJM1-F & CGCTAGCGCTACCGGATGGCCCTGGATCAGCGC & Cloning \\
\hline 4 & 66-LJM1-R & TCGAGGTCGAGAATITAAAACGGGCCCCTTCTCG & Cloning \\
\hline 5 & 30-LJM1-F & CGCTAGCGCTACCGGATGGGTGAGCCAGTGGATCC & Cloning \\
\hline 6 & 30-LJM1-R & TCGAGGTCGAGAATITTAAACGGGCCCCTTCTCG & Cloning \\
\hline 7 & 24-LJM1-F & CGCTAGCGCTACCGGATGGCAGCGCTCGAGGGC & Cloning \\
\hline 8 & 24-LJM1-R & TCGAGGTCGAGAATITTAAACGGGCCCCTTGTCGTC & Cloning \\
\hline 9 & ORF66 1-200 F Nstrep & GAGAAGGGGGCGGCCGCCCTGGATCAGCGCTGG & Cloning \\
\hline 10 & ORF66 1-200 R Nstrep & GCCCTCTAGACTCGATTATGCCGCCCCTATCCCG & Cloning \\
\hline 11 & ORF66 200-429 F & TACCGAGCTCGGATCATGGCAGATACCGGCCTCCGG & Cloning \\
\hline 12 & ORF66 200-429 R & CACCGCCTCCCTCGACGGAGGAACACTTCCCGC & Cloning \\
\hline 13 & $66-C 244 A-F$ & GCCGAGCAGGAGGGACCTGGTCTCGTGCGGAAC & Inverse PCR site-directed mutagenesis \\
\hline 14 & $66-C 244-\mathrm{R}$ & GGCGGGCAGCACTACGTGGCGGGACTTAAT & Inverse PCR site-directed mutagenesis \\
\hline 15 & $66-C 289 A-F$ & GCCGCTCGGGCGGTTGTGTGCATGGAGTGTGGA & Inverse PCR site-directed mutagenesis \\
\hline 16 & 66-C289-R & ACGCGCCCGCTTGGCAAGGGCCGCGCGGGC & Inverse PCR site-directed mutagenesis \\
\hline 17 & $66-C 295 A-F$ & GCCATGGAGTGTGGACACTGTCTTAACTITGGC & Inverse PCR site-directed mutagenesis \\
\hline 18 & $66-C 295-R$ & CACAACCGCCCGAGCGCAACGCGCCCGCTT & Inverse PCR site-directed mutagenesis \\
\hline 19 & $66-C 298 A-F$ & GCCGGACACTGTCTTAACTITGGCAGGGGCAAG & Inverse PCR site-directed mutagenesis \\
\hline 20 & $66-C 298-R$ & CTCCATGCACACAACCGCCCGAGCGCAACG & Inverse PCR site-directed mutagenesis \\
\hline 21 & $66-C 301 A-F$ & GCCCTTAACTTTGGCAGGGGCAAGTTTCATACT & Inverse PCR site-directed mutagenesis \\
\hline 22 & $66-C 301-R$ & GTGTCCACACTCCATGCACACAACCGCCCG & Inverse PCR site-directed mutagenesis \\
\hline 23 & $66-C 341 A-F$ & GCCTCTTACTGTGGCAGCGAACATATGAGGGTG & Inverse PCR site-directed mutagenesis \\
\hline 24 & $66-C 341-R$ & GTAGATCCTCCCCGTGGTCGCACAGATGGT & Inverse PCR site-directed mutagenesis \\
\hline 25 & $66-C 344 A-F$ & GCCGGCAGCGAACATATGAGGGTGTATCCCCTG & Inverse PCR site-directed mutagenesis \\
\hline 26 & $66-C 344-R$ & GTAAGAACAGTAGATCCTCCCCGTGGTCGC & Inverse PCR site-directed mutagenesis \\
\hline 27 & $66-C 355 A-F$ & GCCGATATTACCGGACGCGGGACCCTAGCACGC & Inverse PCR site-directed mutagenesis \\
\hline 28 & $66-C 355-R$ & CAGGGGATACACCCTCATATGTTCGCTGCC & Inverse PCR site-directed mutagenesis \\
\hline 29 & $66-C 393 A-F$ & GCCCTTGGGACGCCCGACTGCGAGGCTGCCCTA & Inverse PCR site-directed mutagenesis \\
\hline 30 & $66-C 393-R$ & AGGCACTACAAAACTGACAGTTTGATCTAG & Inverse PCR site-directed mutagenesis \\
\hline 31 & $66-C 399 A-F$ & GCCGAGGCTGCCCTATTAAAGCACCGTGACGTG & Inverse PCR site-directed mutagenesis \\
\hline 32 & 66-C399-R & GTCGGGCGTCCCAAGGCAAGGCACTACAAAAC & Inverse PCR site-directed mutagenesis \\
\hline 33 & $66-C 423 A-F$ & GCCTGCGGGAAGTGTTCCTCCCTCGAGGGAGGC & Inverse PCR site-directed mutagenesis \\
\hline 34 & $66-C 423-R$ & GAACTCCAGCAGCTGTGAGGTGAGCTGAAG & Inverse PCR site-directed mutagenesis \\
\hline 35 & $66-C 424 A-F$ & GCCGGGAAGTGTTCCTCCCTCGAGGGAGGCGGT & Inverse PCR site-directed mutagenesis \\
\hline 36 & $66-C 424-R$ & ACAGAACTCCAGCAGCTGTGAGGTGAGCTG & Inverse PCR site-directed mutagenesis \\
\hline 37 & $66-C 427 A-F$ & GCCTCCTCCCTCGAGGGAGGCGGTGGATGGAGC & Inverse PCR site-directed mutagenesis \\
\hline 38 & $66-C 427-R$ & СTTCCCGCAACAGAACTCCAGCAGCTGTGA & Inverse PCR site-directed mutagenesis \\
\hline 39 & ORF59Pr_qPCR_F & AATCCACAGGCATGATTGC & DNA replication qPCR \\
\hline 40 & ORF59Pr_qPCR_R & САСАСТТССАССТССССТАА & DNA replication qPCR \\
\hline 41 & GAPDHPr_qPCR-F & TACTAGCGGTITACGGGCG & DNA replication qPCR \\
\hline 42 & GAPDHPr_qPCR-R & TCGAACAGGAGGAGCAGAGACCGA & DNA replication qPCR \\
\hline 43 & K8.1CDS_qPCR_F & CCGTCGGTGTGTAGGGATAAAG & RT-qPCR \\
\hline 44 & K8.1CDS_qPCR_R & GTCGTTGTAGTGGTGGCAGAAA & RT-qPCR \\
\hline 45 & ORF68-CDS-qPCR_F & CCCTGGCAACTCGGTACAAT & RT-qPCR \\
\hline 46 & ORF68-CDS-qPCR_R & AAGATGGTTGGAGCGACTGG & RT-qPCR \\
\hline 47 & 18sCDS_qPCR_F & GTAACCCGTTGAACCCCATT & RT-qPCR \\
\hline 48 & 18sCDS_qPCR_R & CCATCCAATCGGTAGTAGCG & RT-qPCR \\
\hline 49 & K8.1Pr-F & GGGAGAACCATGCCAGACTTTG & ChIP-qPCR \\
\hline 50 & K8.1Pr-R & GCATAGGATTAGGAGCGCCAC & ChIP-qPCR \\
\hline 51 & ORF37Pr-F & GCTGGCGTITCAGAAGCAGTG & ChIP-qPCR \\
\hline 52 & ORF37Pr-R & CCATCCAGGGTGTCAACCA & ChIP-qPCR \\
\hline
\end{tabular}

QuantStudio3 real-time PCR machine with primers for the KSHV ORF59 promoter and normalized to the level of the GAPDH (glyceraldehyde-3-phosphate dehydrogenase) promoter (Table 1).

Infectious virion production was determined by a supernatant transfer assay. Supernatant from induced iSLK cells was syringe filtered through a $0.45-\mu \mathrm{m}$-pore-size filter, and then $2 \mathrm{ml}$ of the supernatant was spinoculated onto $1 \times 10^{6}$ freshly trypsinized HEK293T cells for $2 \mathrm{~h}$ at 1,000 $\times \mathrm{g}$. After $24 \mathrm{~h}$, the medium was aspirated, and the cells were washed once with cold phosphate-buffered saline (PBS) and cross-linked in 4\% paraformaldehyde (Electron Microscopy Services) diluted in PBS. The cells were pelleted and resuspended in PBS, and 50,000 cells/sample were analyzed on a BD Accuri 6 flow cytometer. The data were analyzed using FlowJo software (version 10).

Total RNA and protein were isolated from reactivated iSLK cells at $72 \mathrm{~h}$. RNA was isolated using a Direct-Zol RNA miniprep plus kit (Zymo). Purified RNA was treated with Turbo DNase (Thermo Fisher), and then cDNA was synthesized using avian myeloblastosis virus reverse transcriptase (Promega). The cDNA was used for qPCR analysis using iTaq Universal SYBR green supermix (Bio-Rad), and signals for 
each ORF were normalized to the signal for $18 \mathrm{~S}$ rRNA. Protein samples were resuspended in lysis buffer (150 mM NaCl, $50 \mathrm{mM}$ Tris-HCl, pH 7.4, 1 mM EDTA, 0.5\% NP-40, and protease inhibitor [Roche]), rotated for $30 \mathrm{~min}$ at $4^{\circ} \mathrm{C}$, and clarified by centrifugation at $21,000 \times g$ for $10 \mathrm{~min}$, and then $25 \mu \mathrm{g}$ of lysate was used for SDS-PAGE and Western blotting in Tris-buffered saline and $0.2 \%$ Tween 20 (TBST) using rabbit anti-K8.1 (1:10,000), rabbit anti-ORF59 (1:10,000), rabbit anti-ORF6 (1:10,000), rabbit anti-ORF68 $(1: 5,000)$, mouse anti-ORF26 (1:500; Novus), and mouse anti-GAPDH (1:1,000; Abcam). Rabbit anti-ORF59, anti-K8.1 serum, and anti-ORF6 serum were produced by the Pocono Rabbit Farm and Laboratory by immunizing rabbits against full-length maltose-binding protein (MBP)-ORF59, MBP-K8.1, or MBP-ORF6 (gifts from Denise Whitby [24]). Rabbit anti-ORF68 was previously described (25).

Immunoprecipitation and Western blotting. For all DNA transfections, HEK293T cells were plated and transfected after $24 \mathrm{~h}$ at $\sim 70 \%$ confluence with the PolyJet reagent (SignaGen). Cell lysates were prepared at $24 \mathrm{~h}$ after transfection by washing and pelleting cells in cold PBS, followed by resuspension in lysis buffer and rotation at $4^{\circ} \mathrm{C}$ for $30 \mathrm{~min}$. For isolation of endogenous HA-tagged proteins, $3.5 \times 10^{6}$ iSLK cells (iSLK-BAC16 cell lines ORF66-HA, ORF66-HA/ORF24.stop, HA-ORF24, HA-ORF24/ORF66.stop, and HA-ORF24/ORF30.stop) were reactivated for $48 \mathrm{~h}$ with $5 \mu \mathrm{g} / \mathrm{ml}$ doxycycline and $1 \mathrm{mM}$ sodium butyrate. Lysates were clarified by centrifugation at $21,000 \times g$ for $10 \mathrm{~min}$, and then $1 \mathrm{mg}$ (for pairwise interaction immunoprecipitations [IPs]), 1.5 to $2 \mathrm{mg}$ (for the entire late gene complex IPs), or $1.5 \mathrm{mg}$ (for HA-tagged ORF66 and ORF24) of lysate was incubated with prewashed MagStrep type 3 XT beads (IBA Lifesciences) (for pairwise interaction IPs), M2 anti-FLAG magnetic beads (Sigma) (for the entire late gene complex IPs), or anti-HA magnetic beads (Pierce) overnight in $150 \mathrm{mM} \mathrm{NaCl}, 50 \mathrm{mM}$ Tris- $\mathrm{HCl}, \mathrm{pH}$ 7.4. The beads were washed 3 times for 5 min each time with IP wash buffer $(150 \mathrm{mM} \mathrm{NaCl}, 50 \mathrm{mM}$ Tris-HCl, pH 7.4, $0.05 \%$ NP-40) and eluted with $2 \times$ Laemmli sample buffer (Bio-Rad). Lysates and elutions were resolved by SDS-PAGE and underwent Western blot analysis in TBST using the following primary antibodies: Strep-horseradish peroxidase (HRP) (1:2,500; Millipore), rabbit anti-FLAG (1:3,000; Sigma), mouse anti-FLAG (1:1,000; Sigma), rabbit anti-vinculin (1:1,000; Abcam), mouse anti-Pol II C-terminal domain clone 8WG16 (1:1,000; Abcam), or rabbit anti-HA (1:1,000; Cell Signaling). Following incubation with primary antibodies, the membranes were washed with TBST and incubated with the appropriate secondary antibody. The secondary antibodies used were the following: goat anti-mouse immunoglobulin-HRP (1:5,000; Southern Biotech) or goat anti-rabbit immunoglobulin-HRP (1:5,000; Southern Biotech).

Late gene reporter assay. For assays in HEK293T cells, $1 \times 10^{6}$ cells were plated in 6-well plates, and after $24 \mathrm{~h}$, each well was transfected with $900 \mathrm{ng}$ of DNA containing $125 \mathrm{ng}$ each of pCDNA4/TO ORF18-2xStrep, ORF24-2xStrep, ORF30-2xStrep, ORF31-2xStrep, 2xStrep-ORF34, or wild-type or mutant ORF66-2xStrep (or, as a control, $750 \mathrm{ng}$ of empty pcDNA4/TO-2xStrep plasmid) with either K8.1 Pr pGL4.16 or ORF57 Pr pGL4.16, along with $25 \mathrm{ng}$ of pRL-TK as an internal transfection control. For assays in iSLK cells, $5 \times 10^{5}$ iSLK-ORF66.stop cells were plated in 6-well plates, and after $24 \mathrm{~h}$, each well was reactivated with $5 \mu \mathrm{g} / \mathrm{ml}$ doxycycline and $1 \mathrm{mM}$ sodium butyrate, followed immediately by transfection with $500 \mathrm{ng}$ wild-type or mutant pCDNA4/TO-ORF66-2xStrep (or, as a control, $500 \mathrm{ng}$ of empty pCDNA4/ TO-2xStrep plasmid), $475 \mathrm{ng} \mathrm{K8.1} \mathrm{Pr} \mathrm{pGL4.16+Ori,} \mathrm{and} 25 \mathrm{ng}$ pRL-TK. After $24 \mathrm{~h}$ (for HEK293T cell assays) or $48 \mathrm{~h}$ (for iSLK cell assays), the cells were rinsed twice with PBS, lysed by rocking for $15 \mathrm{~min}$ at room temperature in $500 \mu$ l of passive lysis buffer (Promega), and clarified by centrifugation at $21,000 \times g$ for $2 \mathrm{~min}$. Twenty microliters of the clarified lysate was added in triplicate to a white chimney well microplate (Greiner Bio-One) to measure luminescence on a Tecan M1000 microplate reader using a dual-luciferase assay kit (Promega). The firefly luminescence was normalized to that of the internal renilla luciferase control for each transfection. The results for all samples were normalized to those for the corresponding control containing empty plasmid.

ChIP. Chromatin immunoprecipitation (ChIP) was performed on 15-cm plates of iSLK cells (iSLKBAC16 cell lines WT, ORF66-HA, ORF66-HA/ORF24.stop, HA-ORF24, HA-ORF24/ORF66.stop, and HAORF24/ORF30.stop) that had been reactivated for $48 \mathrm{~h}$ with $5 \mu \mathrm{g} / \mathrm{ml}$ doxycycline and $1 \mathrm{mM}$ sodium butyrate. The cells were cross-linked in $2 \%$ formaldehyde for $10 \mathrm{~min}$ at room temperature, quenched in $0.125 \mathrm{M}$ glycine for $5 \mathrm{~min}$, and washed twice with ice-cold PBS. Cross-linked cell pellets were mixed with $1 \mathrm{ml}$ ice-cold ChIP lysis buffer (50 mM HEPES, pH 7.9, $140 \mathrm{mM} \mathrm{NaCl}, 1 \mathrm{mM}$ EDTA, 10\% glycerol, 0.5\% NP-40, $0.25 \%$ Triton X-100, protease inhibitor [Roche]), incubated by rotation at $4^{\circ} \mathrm{C}$ for $10 \mathrm{~min}$, and then spun at $1,700 \times g$ for $5 \mathrm{~min}$ at $4^{\circ} \mathrm{C}$. The nuclei were resuspended in wash buffer $(10 \mathrm{mM} \mathrm{Tris}-\mathrm{HCl}, \mathrm{pH} 7.5$, $100 \mathrm{mM} \mathrm{NaCl}, 1 \mathrm{mM}$ EDTA pH 8.0, protease inhibitor [Roche]) and rotated for $10 \mathrm{~min}$ at $4^{\circ} \mathrm{C}$. The nuclei were collected by centrifugation at $1,700 \times g$ for $5 \mathrm{~min}$ at $4^{\circ} \mathrm{C}$ and then gently rinsed with shearing buffer ( $50 \mathrm{mM}$ Tris-HCl, pH 7.5, $10 \mathrm{mM}$ EDTA, $0.1 \%$ SDS), followed by centrifugation at $1,700 \times g$ for $5 \mathrm{~min}$ at $4^{\circ} \mathrm{C}$. After a second rinse with shearing buffer, the nuclei were resuspended in $1 \mathrm{ml}$ of shearing buffer and transferred to a milliTube with AFA fiber (Covaris). Chromatin was sheared using a Covaris S220 ultrasonicator for 5 min (peak power, 140; duty cycle, 5; number of cycles/burst, 200).

The chromatin was spun at $16,000 \times g$ for $10 \mathrm{~min}$ at $4^{\circ} \mathrm{C}$, and the pellet was discarded. The chromatin was precleared with protein A-protein G beads blocked with $200 \mu \mathrm{g} / \mathrm{ml}$ glycogen, $200 \mu \mathrm{g} / \mathrm{ml}$ bovine serum albumin, $200 \mu \mathrm{g} / \mathrm{ml} \mathrm{E}$. coli tRNA for $2 \mathrm{~h}$ at $4^{\circ} \mathrm{C}$. Precleared chromatin $(25 \mu \mathrm{g})$ was diluted in shearing buffer to $500 \mu \mathrm{l}$, adjusted to include $150 \mathrm{mM} \mathrm{NaCl}$ and $1 \%$ Triton X-100, and then incubated with $10 \mu \mathrm{g}$ anti-HA antibody (catalog number C29F4; Cell Signaling) or $10 \mu \mathrm{g}$ rabbit IgG (Southern Biotech) overnight. Samples were rotated with $25 \mu \mathrm{l}$ preblocked protein A-protein $\mathrm{G}$ beads (Thermo Fisher) for $2 \mathrm{~h}$ at $4^{\circ} \mathrm{C}$. The beads were washed with low-salt immune complex ( $20 \mathrm{mM}$ Tris, pH 8.0, $1 \%$ Triton X-100, 2 mM EDTA, $150 \mathrm{mM} \mathrm{NaCl}, 0.1 \%$ SDS), high-salt immune complex (20 mM Tris, pH 8.0, 1\% Triton X-100, $2 \mathrm{mM}$ EDTA, $500 \mathrm{mM} \mathrm{NaCl}, 0.1 \% \mathrm{SDS}$ ), lithium chloride immune complex (10 mM Tris, $\mathrm{pH}$ 8.0, $0.25 \mathrm{M} \mathrm{LiCl}, 1 \% \mathrm{NP}-40,1 \%$ deoxycholic acid, $1 \mathrm{mM}$ EDTA), and TE buffer (10 mM Tris-HCl, pH 8.0, $1 \mathrm{mM}$ 
EDTA) for $10 \mathrm{~min}$ each at $4^{\circ} \mathrm{C}$ with rotation. DNA was eluted from the beads using $100 \mu \mathrm{l}$ of elution buffer $(150 \mathrm{mM} \mathrm{NaCl}, 50 \mu \mathrm{g} / \mathrm{ml}$ proteinase $\mathrm{K})$ and incubated at $55^{\circ} \mathrm{C}$ for $2 \mathrm{~h}$ and then at $65^{\circ} \mathrm{C}$ for $12 \mathrm{~h}$. DNA was purified using an Oligo Clean \& Concentrator kit (Zymo Research). Purified DNA was quantified by qPCR using iTaq Universal SYBR Mastermix (Bio-Rad) and the primers indicated in Table 1 for 50 cycles. Each sample was normalized to its own input.

\section{ACKNOWLEDGMENTS}

We thank Divya Nandakumar for her helpful suggestions and critical reading of the manuscript. We thank Matthew Gardner for cloning the ORF66.stop and mutant rescue BACs.

A.L.D. is The Rhee Family Fellow of the Damon Runyon Cancer Research Foundation (DRG-2349-18). B.A.G. is an investigator of the Howard Hughes Medical Institute. This research was also supported by NIH grant R01AI122528 to B.A.G.

\section{REFERENCES}

1. Nandakumar D, Glaunsinger B. 2019. An integrative approach identifies direct targets of the late viral transcription complex and an expanded promoter recognition motif in Kaposi's sarcoma-associated herpesvirus. PLoS Pathog 15:e1007774. https://doi.org/10.1371/journal.ppat.1007774.

2. Tang S, Yamanegi K, Zheng ZM. 2004. Requirement of a 12-base-pair TATT-containing sequence and viral lytic DNA replication in activation of the Kaposi's sarcoma-associated herpesvirus K8.1 late promoter. J Virol 78:2609-2614. https://doi.org/10.1128/jvi.78.5.2609-2614.2004.

3. Wong-Ho E, Wu TT, Davis ZH, Zhang B, Huang J, Gong H, Deng H, Liu F, Glaunsinger B, Sun R. 2014. Unconventional sequence requirement for viral late gene core promoters of murine gammaherpesvirus 68. J Virol 88:3411-3422. https://doi.org/10.1128/JVI.01374-13.

4. Aubry V, Mure F, Mariame B, Deschamps T, Wyrwicz LS, Manet E, Gruffat H. 2014. Epstein-Barr virus late gene transcription depends on the assembly of a virus-specific preinitiation complex. J Virol 88 12825-12838. https://doi.org/10.1128/JVI.02139-14.

5. Davis ZH, Hesser CR, Park J, Glaunsinger BA. 2016. Interaction between ORF24 and ORF34 in the Kaposi's sarcoma-associated herpesvirus late gene transcription factor complex is essential for viral late gene expression. J Virol 90:599-604. https://doi.org/10.1128/JVI.02157-15.

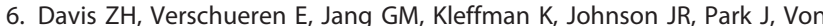
Dollen J, Maher MC, Johnson T, Newton W, Jager S, Shales M, Horner J, Hernandez RD, Krogan NJ, Glaunsinger BA. 2015. Global mapping of herpesvirus-host protein complexes reveals a transcription strategy for late genes. Mol Cell 57:349-360. https://doi.org/10.1016/j.molcel.2014 .11 .026 .

7. Gruffat $H$, Marchione R, Manet E. 2016. Herpesvirus late gene expression: a viral-specific pre-initiation complex is key. Front Microbiol 7:869. https://doi.org/10.3389/fmicb.2016.00869.

8. Brulois K, Wong LY, Lee HR, Sivadas P, Ensser A, Feng P, Gao SJ, Toth Z, Jung JU. 2015. Association of Kaposi's sarcoma-associated herpesvirus ORF31 with ORF34 and ORF24 is critical for late gene expression. J Virol 89:6148-6154. https://doi.org/10.1128/JVI.00272-15.

9. Gong D, Wu NC, Xie Y, Feng J, Tong L, Brulois KF, Luan H, Du Y, Jung JU, Wang CY, Kang MK, Park NH, Sun R, Wu TT. 2014. Kaposi's sarcomaassociated herpesvirus ORF18 and ORF30 are essential for late gene expression during lytic replication. J Virol 88:11369-11382. https://doi .org/10.1128/JVI.00793-14.

10. Nishimura M, Watanabe T, Yagi S, Yamanaka T, Fujimuro M. 2017. Kaposi's sarcoma-associated herpesvirus ORF34 is essential for late gene expression and virus production. Sci Rep 7:329. https://doi.org/10.1038/ s41598-017-00401-7.

11. Castaneda AF, Glaunsinger BA. 2019. The interaction between ORF18 and ORF30 is required for late gene expression in Kaposi's sarcomaassociated herpesvirus. J Virol 93:e01488-18. https://doi.org/10.1128/JVI .01488-18.

12. Pan D, Han T, Tang S, Xu W, Bao Q, Sun Y, Xuan B, Qian Z. 2018. Murine cytomegalovirus protein pM91 interacts with pM79 and is critical for viral late gene expression. J Virol 92:e00675-18. https://doi.org/10.1128/ JVI.00675-18.
13. Wyrwicz LS, Rychlewski L. 2007. Identification of herpes TATT-binding protein. Antiviral Res 75:167-172. https://doi.org/10.1016/j.antiviral.2007 .03 .002 .

14. Didychuk AL, Castañeda AF, Kushnir LO, Huang CJ, Glaunsinger BA. 2020. Conserved CxnC motifs in Kaposi's sarcoma-associated herpesvirus ORF66 are required for viral late gene expression and mediate its interaction with ORF34. bioRxvi https://doi.org/10.1101/728139.

15. Brulois KF, Chang H, Lee AS, Ensser A, Wong LY, Toth Z, Lee SH, Lee HR, Myoung J, Ganem D, Oh TK, Kim JF, Gao SJ, Jung JU. 2012. Construction and manipulation of a new Kaposi's sarcoma-associated herpesvirus bacterial artificial chromosome clone. J Virol 86:9708-9720. https://doi .org/10.1128/JVI.01019-12.

16. Martinez-Guzman D, Rickabaugh T, Wu TT, Brown H, Cole S, Song MJ, Tong L, Sun R. 2003. Transcription program of murine gammaherpesvirus 68. J Virol 77:10488-10503. https://doi.org/10.1128/jvi.77.19.10488 $-10503.2003$.

17. Summers WC, Klein G. 1976. Inhibition of Epstein-Barr virus DNA synthesis and late gene expression by phosphonoacetic acid. J Virol 18: $151-155$.

18. Chakravorty A, Sugden B, Johannsen EC. 2019. An epigenetic journey: Epstein-Barr virus transcribes chromatinized and subsequently unchromatinized templates during its lytic cycle. J Virol 93:e02247-18. https:// doi.org/10.1128/JVI.02247-18

19. Notredame C, Higgins DG, Heringa J. 2000. T-Coffee: a novel method for fast and accurate multiple sequence alignment. J Mol Biol 302:205-217. https://doi.org/10.1006/jmbi.2000.4042.

20. Laity JH, Lee BM, Wright PE. 2001. Zinc finger proteins: new insights into structural and functional diversity. Curr Opin Struct Biol 11:39-46. https://doi.org/10.1016/S0959-440X(00)00167-6.

21. Chen Y, Ciustea M, Ricciardi RP. 2005. Processivity factor of KSHV contains a nuclear localization signal and binding domains for transporting viral DNA polymerase into the nucleus. Virology 340:183-191. https:// doi.org/10.1016/j.virol.2005.06.017.

22. Gruffat H, Kadjouf F, Mariame B, Manet E. 2012. The Epstein-Barr virus BcRF1 gene product is a TBP-like protein with an essential role in late gene expression. J Virol 86:6023-6032. https://doi.org/10.1128/JVI .00159-12.

23. Basehoar AD, Zanton SJ, Pugh BF. 2004. Identification and distinct regulation of yeast TATA box-containing genes. Cell 116:699-709. https://doi.org/10.1016/s0092-8674(04)00205-3.

24. Labo N, Miley W, Marshall V, Gillette W, Esposito D, Bess M, Turano A, Uldrick T, Polizzotto MN, Wyvill KM, Bagni R, Yarchoan R, Whitby D. 2014 Heterogeneity and breadth of host antibody response to KSHV infection demonstrated by systematic analysis of the KSHV proteome. PLoS Pathog 10:e1004046. https://doi.org/10.1371/journal.ppat.1004046.

25. Gardner MR, Glaunsinger BA. 2018. Kaposi's sarcoma-associated herpesvirus ORF68 is a DNA binding protein required for viral genome cleavage and packaging. J Virol 92:e00840-18. https://doi.org/10 $.1128 /$ JVI.00840-18. 